\title{
Development of a risk prediction model for incident hypertension in a working-age Japanese male population
}

Toshiaki Otsuka, Yuko Kachi, Hirotaka Takada, Katsuhito Kato, Eitaro Kodani, Chikao Ibuki, Yoshiki Kusama and Tomoyuki Kawada

Hypertension Research (2015) 38, 445; doi:10.1038/hr.2015.41

Correction to: Hypertension Research (2015) 38, 419-425; doi:10.1038/hr.2014.159; published online 13 November 2014

After online publication of this article, the authors noticed errors in Table 1 and in Supplementary Table 2. Wrong values were presented.
The errors have now been rectified, and the article with correct Table 1 and Supplementary Table 2 appears in this issue. The html and online pdf versions have also been rectified. 